\title{
Do Written Asthma Action Plans Improve Outcomes?
}

\author{
John M. Kelso, MD
}

With appropriate management, children with asthma should expect few symptoms, no limits on activity, rare exacerbations, and normal lung function. Appropriate education of parents and other caregivers of children with asthma has clearly been shown to help achieve these goals. Although recommended in asthma guidelines, providing written asthma action plans does not improve outcomes beyond asthma education alone.

\section{Introduction}

$\mathrm{T}$ HE GOAL OF ASTHMA management is "reducing the symptoms, functional limitations, impairment in quality of life, and risk of adverse events that are associated with the disease." 1 As with any chronic disease, parents and other caregivers of children with asthma should know how to care for their child's condition. In the case of asthma, this would include "(1) daily management and (2) how to recognize and handle worsening asthma." 1 Thus, clinicians need to provide parents with this information. But does it need to be provided in the form of a written asthma action plan?

The National Heart, Lung, and Blood Institute (NHLBI) National Asthma Education and Prevention Program (NAEPP) Expert Panel Report 3: Guidelines for the Diagnosis and Management of Asthma was published in 2007. ${ }^{1}$ The guidelines recommend that all children with asthma have a written asthma action plan and that one be provided to the child's school. ${ }^{1}$ However, this recommendation is based on studies involving mostly adult patients. Furthermore, the recommendation is given an evidence category of $\mathrm{B}$, described as being based on a "limited body of data." 1 The uncertainty of the effectiveness of written asthma action plans is reflected in the report's statement that "although the results of these studies are mixed, they suggest that the use of written plans may help patients improve control of their asthma.",

The Global Initiative for Asthma (GINA) 2015 update also recommends that all patients with asthma be provided with a written asthma action plan, indicating that this recommendation has an evidence category of $\mathrm{A}$, described as being based on a "rich body of data.,"2 GINA states that "the essential components of effective guided asthma selfmanagement include self-monitoring of symptoms and/or peak flow, a written asthma action plan to show how to recognize and respond to worsening asthma and regular review of asthma control, treatment and skills by a healthcare provider," but most of the evidence provided is based on adult studies and does not separate out these components, that is, does not evaluate whether the written asthma action plan is a required element. ${ }^{2}$ The document does state that "less intensive interventions that involve self-management education but not a written action plan are less effective", ; however, the reference cited to support this claim specifically states that "optimal self-management allowing for optimisation of asthma control by adjustment of medications may be conducted by either self-adjustment with the aid of a written action plan or by regular medical review.",3

This review will examine the evidence regarding the effectiveness of written asthma action plans beyond asthma education alone in children.

\section{What Is Meant by "Effective"?}

If we want to determine whether or not written asthma action plans are effective, we first need to define effectiveness. If written asthma action plans are effective, they should help achieve the goals of asthma therapy stated above, namely "reducing the symptoms, functional limitations, impairment in quality of life, and risk of adverse events that are associated with the disease." Potential outcome measures regarding symptoms could include symptom frequency, particularly those requiring use of a rescue medication, typically albuterol. Assessment of functional limitations could include missed school days or limits on physical activity. Quality of life assessment could be evaluated with questionnaires specifically designed for this purpose. Perhaps the most important assessment would be the risk of adverse events, particularly exacerbations requiring systemic corticosteroids, unscheduled visits or hospitalizations, or permanent decline in lung function or "airway remodeling."

\section{Asthma Education Versus a Written Asthma Action Plan}

All parents of children with asthma need education about the child's disease and how to treat it. This typically

Division of Allergy, Asthma and Immunology, Scripps Clinic, San Diego, California.

(C) John M. Kelso 2016; Published by Mary Ann Liebert, Inc. This Open Access article is distributed under the terms of the Creative Commons Attribution Noncommercial License (http://creativecommons.org/licenses/by-nc/4.0/) which permits any noncommercial use, distribution, and reproduction in any medium, provided the original author(s) and the source are credited. 
involves a discussion with the parents about the pathophysiology of asthma and the use of controller and rescue medications, including education on appropriate medication delivery devices, such as metered dose inhalers with spacers, dry powder inhalers, and nebulizers. This discussion would also include the expectations of treatment, namely that with effective controller therapy, rescue albuterol should be needed infrequently and effective when it is needed to relieve symptoms or prevent exercise-induced symptoms, physical activity or sports need not be limited in any way, and unscheduled visits or need for systemic corticosteroids should be rare and hospitalizations rarer still. Lung function should be normal and maintained in both the short and long terms. The parents also need to know what to do and how to contact the provider if these expectations are not being met. Does providing a written asthma action plan convey this information in a more effective way (defined above) than simply explaining it to the parents? To answer this question, we need to examine studies that evaluate not asthma education versus no education but rather asthma education with or without provision of a written asthma action plan.

Asthma action plans are typically divided into colorcoded zones, which call for various actions based on the child's current symptoms or peak flow measurements. ${ }^{4}$ Studies have shown equivalent outcomes whether the action plan is based on symptoms or peak flow measurements. ${ }^{1,2}$ Patients in the "green zone" (no symptoms) are instructed to continue to take their controller medications. ${ }^{4}$ Patients in the "yellow zone" (having symptoms) are instructed to use several doses of albuterol and if not improving to take oral corticosteroids or call their doctor." Patients in the "red zone" (having symptoms unresponsive to albuterol) are instructed to use several doses of albuterol and take oral corticosteroids and call their doctor. ${ }^{4}$ This information, despite being organized into color-coded "zones," does not really convey any additional information to the parents beyond that provided in the asthma education described above. The parents would have been told which medications are controller medications, instructed in their use, and told the importance of their regular use even if no symptoms are present. Specific dosing instructions are also written on the medication containers themselves. In many clinical settings, patients are also provided with a list of their current medications after each office visit. The parents would also have been told that albuterol is for the relief of symptoms and should provide a prompt response and that additional doses can be given if necessary, but their need indicates worsening asthma. Based on the particular child's history, a small subset of parents will have oral corticosteroids available at home, which can be used for an exacerbation. All parents of children with asthma should be able to contact their child's provider or another on-call provider to discuss additional measures for asthma symptoms unresponsive to albuterol. In addition to this education, does providing a written asthma action plan lead to better asthma outcomes?

\section{The Evidence}

In 2005, Agrawal et al. conducted a prospective, open, randomized parallel-group controlled trial involving 60 children between the ages of 5 and 12 years with physiciandiagnosed moderate persistent asthma per the NHLBI guidelines. ${ }^{5}$ All children were receiving moderate-dose inhaled corticosteroids regularly and inhaled beta- 2 agonist as needed. At the time of enrollment, all patients and parents were given education about asthma and its causes, aggravating factors, purpose, and effects of asthma therapy and the principles of home monitoring and self-management of asthma. All were asked to keep a diary recording daytime or nighttime cough, wheezing, difficulty in breathing, missing of school, exercise intolerance, and use of rescue medication. They were then randomized into 2 groups: one group receiving individualized written home management plans and another group not receiving such written asthma action plans. Subjects were followed up every week for the first month and then monthly for the next 3 months. At each visit, the following were recorded: number of acute asthma events (defined as a clinical situation necessitating an emergency hospital visit, hospital admission, or unscheduled doctor visit), number of school days missed, number of nighttime awakenings with symptoms, and symptom score. Children receiving the written home management plan had significantly fewer acute asthma events [mean (SD) 0.50 (0.71) versus $1.0(0.61), P=0.02]$, loss of school days [1.5 (1.4) versus 2.54 (1.79), $P=0.015$ ], nocturnal awakenings [1.75 (1.30) versus $3.25(1.20), P=0.001]$, and better overall symptom scores [21.9 (14.4) versus 33.7 (10.9), $P=0.0006]$. The authors concluded that "the addition of a written individualized home management plan improves the overall control of asthma in children with moderate persistent disease severity and should form an integral component of treatment protocols in addition to family education and pharmacological interventions."

In 2009, Espinoza-Palma et al. conducted a prospective study of 77 children 5 to 15 years of age admitted to the hospital for asthma. ${ }^{6}$ All parents completed a questionnaire about their child's asthma in the previous 12 months. All families received education about the etiology, triggers, types, severity and treatment of asthma, and the correct use of the inhalers with spacers. They were then randomized to receive a written self-management plan or not. All families were asked to record any asthma exacerbations, hospitalizations, emergency visits, and the therapy used in exacerbations, as well as chronic therapy used monthly for 1 year. Asthma exacerbations were defined as the use of rescue bronchodilators for more than 2 days. They were contacted by telephone every 3 months for a year by an external source blinded to this study group to collect the information recorded by the parents. The primary outcome was number of exacerbations during the study year. The secondary outcomes included rehospitalizations, prednisone bursts, and emergency visits. At the end of the 1-year follow-up, there were significant decreases in exacerbations (51\% decline), prednisone bursts (62\%), emergency visits (60\%), and hospitalizations $(75 \%)$ in both groups; however, there were no differences between the groups. The authors concluded that "asthma education with or without a self-management plan during asthma hospitalization was effective."

In 2011, Sunshine et al. conducted a 12-month study involving 251 children aged 3 to 13 years with persistent or poorly controlled asthma. ${ }^{7}$ All families received standard clinic-based asthma education provided by nurses. Although not randomized, the investigators determined whether or not the children's families had been given written asthma action plans and how often they used them. The primary outcomes 
were asthma control, dichotomized to well controlled (4 or fewer symptom days in the previous 2 weeks) or not well controlled, and a score on the Pediatric Caregiver Quality of Life Scale. The secondary outcome was urgent health services utilization [any self-reported emergency department (ED), hospital, or unscheduled clinic visit in the past 3 months for asthma]. Both groups showed significant increases in the proportion of children who were well controlled (from 59.8\% to $77.6 \%$ among action plan users and from $61.1 \%$ to $77.6 \%$ among nonusers), significant increases in mean quality of life scores (from 5.63 to 6.24 among action plan users and from 5.52 to 6.11 among nonusers), and significant decreases in the proportion of children requiring unscheduled health services (from $49.1 \%$ to $28.5 \%$ among action plan users and from $50.3 \%$ to $23.7 \%$ among nonusers) over 1 year. However, there were no significant differences in baseline-to-exit changes in any of these outcome variables between those who had action plans and those who did not or between those with action plans who used them and those who did not. The authors concluded that "written asthma action plan use during the previous year was not associated with improved outcomes compared with nonuse."

In 2013, Wong et al. conducted a single-blind (physician) randomized controlled trial of 76 children with asthma between the ages of 6 and 17 years. ${ }^{8}$ They were randomly assigned to a study group provided with a symptom-based written asthma action plan or a control group who received only verbal counseling on the management of asthma exacerbations. The children were followed every 3 months over a period of 9 months for a total of 4 visits. At recruitment and at each follow-up, the Asthma Control Test or Child Asthma Control Test (ACT or CACT) and the Standardized Pediatric Asthma Quality of Life Questionnaire (PAQLQ) were administered by an investigator not involved in the clinical assessment of the children. The number of acute asthma exacerbations necessitating unscheduled doctor visits over the previous 3 months was recorded. There were significant improvements in ACT scores in both groups from visit 1 to visit 4, from 16.63 (4.63) to 21.53 (4.25) [mean (SD)] in the control group $(P=0.003)$ and from 20.38 (3.26) to 23.12 (2.23) in the action plan group $(P=0.008)$, but there was no change in quality of life scores or number of asthma exacerbations in either group. However, there was no difference in asthma control, quality of life, or asthma exacerbations between the 2 groups. The authors concluded that "the written asthma action plan did not reduce unscheduled doctor visits nor improve asthma control or quality of life."

Finally, in 2015, Sheares et al. conducted a prospective randomized parallel-group controlled trial of 272 children and 135 adults with a physician diagnosis of persistent asthma, as defined by the NAEPP guidelines. ${ }^{9}$ All had been referred to either a pulmonologist or an allergist, and all were new patients who had never been seen by a subspecialist physician for asthma care and had never received a written asthma action plan. All received asthma education provided by the physician. They were randomized to receive a written asthma action plan or not. The primary outcomes included asthma symptom frequency measured in 3 ways (average number of days with asthma symptoms, average number of nights with symptoms, and average number of days of short-acting bronchodilator use), ED visits (assessed using 3-month recall), and asthma quality of life [using the Juniper Mini Asthma Quality of Life Questionnaire (MiniAQLQ) for adult participants and the Pediatric Asthma Caregivers Quality of Life Questionnaire (PACQLQ) for pediatric participants]. The secondary outcomes included number of hospitalizations and days with activity limitation. Having a written asthma action plan was not significantly associated with a reduction in days with symptoms [relative risk (RR), 1.01; 95\% confidence interval (CI), 0.8-1.3; $P=0.94)$, nocturnal symptoms (RR, $0.98 ; 95 \% \mathrm{CI}, 0.7-1.3$; $P=0.9$ ), or short-acting bronchodilator use (RR, 1.00; $95 \%$ CI, 0.8-1.2; $P=0.97$ ) from baseline to 12 months, although both groups showed a significant reduction in all 3 variables $(P=0.0001$ for all). At 12 months, there were significant reductions in ED visits for both groups: action plan group [mean (SD): from 3.3 (5.9) to $1.6(3), P=0.0001]$ and control group [2.5 (3.9) to 1.5 (3), $P=0.0006]$. However, there were no between-group differences in the ED visits. There was a significant increase in mean asthma QoL scores from baseline to 12-month follow-up; however, no betweengroup differences were noted. There were no significant between-group differences in asthma hospitalizations at baseline, and the reduction in admissions was not significant for either group at the 12-month follow-up period. Days with activity limitation declined significantly for both groups: from 3.6 to 2.1 days in the action plan group $(P=0.0005)$ and from 3.9 to 2.3 days in the control subjects $(P=0.0001)$, but there were no significant between-group differences. The authors concluded that "using a written asthma action plan form as a vehicle for providing asthma management instructions to patients with persistent asthma who are receiving subspecialty care for the first time confers no added benefit beyond subspecialty-based medical care and education for asthma."

Thus, 5 studies have examined the use of written asthma action plans in children to determine whether or not the provision of such plans leads to better asthma outcomes beyond those achieved by asthma education alone. All the studies demonstrated that asthma education and regular follow-up care lead to dramatic improvements in outcomes. $^{5-9}$ On the question of whether or not adding a written asthma action plan leads to further improvement beyond that achieved by asthma education alone, the bulk of the evidence clearly says no. Although a study involving 60 children followed for 3 months concluded that the action plan led to better outcomes, ${ }^{5} 4$ subsequent studies involving 676 children followed for up to 1 year all concluded that the action plan did not lead to better outcomes. ${ }^{6-9}$

\section{Is It Possible That Asthma Action Plans Are Actually Harmful?}

A review of several studies found that action plan users had higher rates of hospitalization for asthma, suggesting a possible explanation that "written plans might discourage attendance at the general practitioner or emergency department until the child has deteriorated to a point where hospitalization is inevitable."10 A cross-sectional study evaluating costs and effectiveness of asthma action plans for children determined that the total annual costs of the asthma action plan group were significantly higher than those of the control group despite no difference in health outcomes. ${ }^{11}$ The difference in cost was attributable to greater medication 
and health services use in the action plan group. Thus, although well intentioned, one must consider potential unintended consequences of the use of asthma action plans.

\section{If No Action Plan, Then What?}

At an initial asthma evaluation, time spent developing an asthma action plan (with no proven benefit and possible harm as above) could be better spent providing asthma education (described above), which clearly has benefit, as demonstrated in the studies reviewed here ${ }^{5-9}$ It is helpful to reinforce this education with written materials describing what asthma is, how asthma medications work, and how to use them, with a special emphasis on inhaler technique. At subsequent asthma visits, time spent updating an asthma action plan could be better spent reinforcing asthma education and answering questions. Although parents of children with asthma do not need a written asthma action plan, they do need to know what to do if their child has an increase in asthma symptoms. This "asthma action plan," which can be conveyed verbally, is exactly the same for all patients with asthma and applies equally to both home and school settings. Namely, if the child is having asthma symptoms, they need albuterol. If they are needing the albuterol more frequently or it does not seem to be helping, the healthcare provider should be notified. These 2 sentences encapsulate all the information found on the asthma action plan. It seems reasonable to remind parents of children with asthma of this at every asthma visit, and it takes only seconds to do so.

\section{Conclusion}

Parents of children with asthma need to understand the disease, the medications used to treat it, how to use the medications, what to expect from treatment, and what to do if these expectations are not being met. Providing this asthma education at the time of diagnosis and reinforcing it at all subsequent visits lead to better outcomes in terms of achieving the goals of asthma therapy, namely few symptoms, no limits on activity, rare exacerbations, and normal lung function. ${ }^{5-9}$ Adding a formal written asthma action plan does not lead to better outcomes ${ }^{6-9}$ and may lead to worse outcomes. ${ }^{10,11}$ In developing asthma guidelines, it was not illogical to think that providing written asthma action plans would lead to better outcomes; however, an evidence-based approach says otherwise.

\section{Author Disclosure Statement}

No competing financial interests exist.

\section{References}

1. Expert Panel Report 3. Guidelines for the Diagnosis and Management of Asthma Full Report 2007. National Asthma Education and Prevention Program 2007, National Heart, Lung, and Blood Institute.

2. Global Initiative for Asthma. Global strategy for asthma management and prevention. www.ginasthma.org 2015.

3. Powell H, Gibson PG. Options for self-management education for adults with asthma. Cochrane Database Syst Rev 2003:Cd004107.

4. Asthma Action Plan. NIH Publication No. 07-5251, 2007.

5. Agrawal SK, Singh M, Mathew JL, Malhi P. Efficacy of an individualized written home-management plan in the control of moderate persistent asthma: a randomized, controlled trial. Acta Paediatr 2005; 94:1742-1746.

6. Espinoza-Palma T, Zamorano A, Arancibia F, Bustos MF, Silva MJ, Cardenas C, et al. Effectiveness of asthma education with and without a self-management plan in hospitalized children. J Asthma 2009; 46:906-910.

7. Sunshine J, Song L, Krieger J. Written action plan use in inner-city children: is it independently associated with improved asthma outcomes? Ann Allergy Asthma Immunol 2011; 107:207-213.

8. Wong SS, Nathan AM, de Bruyne J, Zaki R, Mohd Tahir SZ. Does a written asthma action plan reduce unscheduled doctor visits in children? Indian J Pediatr 2013; 80:590595.

9. Sheares BJ, Mellins RB, Dimango E, Serebrisky D, Zhang $\mathrm{Y}$, Bye MR, et al. Do patients of subspecialist physicians benefit from written asthma action plans? Am J Respir Crit Care Med 2015; 191:1374-1383.

10. Burrill R, Carroll W. Towards evidence based medicine for paediatricians. Do written asthma action plans reduce hospital admissions? Arch Dis Child 2009; 94:742-743.

11. Polisena J, Tam S, Lodha A, Laporte A, Coyte PC, Ungar WJ. An economic evaluation of asthma action plans for children with asthma. J Asthma 2007; 44:501-508.

Address correspondence to: John M. Kelso, MD Division of Allergy, Asthma and Immunology Scripps Clinic 3811 Valley Centre Drive San Diego, CA 92130

E-mail: kelso.john@scrippshealth.org

Received for publication January 23, 2016; accepted after revision January 23, 2016. 